\title{
A Brief Review on the Potential of Nanotech in the Defense Sector
}

\author{
Mahidhara Utsav
}

\begin{abstract}
Nanotechnologyrelies on the concept of creating applications based on components built at nanoscale. The field draws on knowledge and expertise from various science disciplines, integrating aspects of physics, chemistry, biology, material sciences, with several fields of engineering. Ever since its beginnings roughly two decades ago, it has evolved into a massive area of interest, attracting attention due to the various possibilities afforded by the size of the nanodevices and their unique properties. Nanoparticles, by virtue of their adaptability have found various applications in fields ranging from biology to defense. This paper deals with the properties of CNTs, nanodevices and nanobots, their applications and the ethical issues concerning their influence in the defense sectors.
\end{abstract}

Index Terms-Battlefield efficiency, defense sector, nanoparticles, nanosensors.

\section{INTRODUCTION}

Technology is developing at an enormous rate. Rapid advancement in this sector has affected every aspect of the world as we know it. From smart phones to nanobots, technical growth has permeated the core of every industry thus rendering it a ubiquitous quality that makes it an inseparable fixture of our daily life. The one sector that has experienced a tectonic shift in its methods of operation due to this is the defense sector.

Defense operations have increasingly become less strategy-oriented and more technology-dominated resulting in a radical change from the traditional methods of warfare. As an example, the change from manned aircrafts to drones has not only resulted in low mortality rates, it has also enhanced the range of operations by being assigned for missions that were otherwise considered too "dull, dirty or dangerous" for manned aircraft [1].

The technological inclination of the defense sector has gradually given way to the proposal and implementation of nanotech-oriented devices ranging from combat suits to weaponry to vehicular protection. Though it can prove advantageous, there are ethical dilemmas to be faced. This paper gives a brief review on the major influence of nanotech on the defense sector and the pros and cons of the enhanced quality of service that it renders.

\section{Properties of All NANOPARTICles}

The proposed nanomaterials considered for the body

Manuscript received November 2, 2013; revised January 15, 2014.

MahidharaUtsav is with BITS Pilani, Dubai Campus Dubai International Academic City, P.O. Box No.: 345055, Dubai, UAE (e-mail: utsav.mahi.210@gmail.com). armours, armament, logistics, vehicles and covert operations include CNTs, nano-structured materials such as silica.

The various properties and their respective applications are:

\section{A. CNT}

CNTs are members of the fullerene structural family. They have long, hollow structures with the walls formed by one-atom-thick sheets of graphene. Nanotubes are categorized as single-walled nanotubes (SWNTs) and multi-walled nanotubes (MWNTs). The carbon atoms of a single (graphene) sheet of graphite form a planar honeycomb lattice, in which each atom is connected via a strong chemical bond to three neighboring atoms.

Ever since their discovery by Sumiolijima in 1991, their various unique properties render them the most sought-after materials for military applications. Their electrical resistance of individual carbon nanotubes is comparable to that predicted for copper wires of the same size, making them the most conductive wires known. Individual carbon nanotubes have follow ballistic conduction (1 D scattering) which permits substantial current flow with minimal heat generation, thus allowing nanotubes to carry enormous amounts of current. Researchers at MIT have confirmed that using CNTs for battery electrodes can produce a tenfold increase in output power of a given weight of material that can be sustained across thousands of charge-discharge cycles, than a conventional lithium-ion battery. The strong covalent bonds formed via honeycomb lattice result in the largest known elastic modulus for any material, hence indicating their high stiffness. Also, they have the ability to endure tension of a weight equivalent to $6422 \mathrm{~kg}$ on a cable with cross-section of $1 \mathrm{~mm}^{2}$ (see Fig. 1).

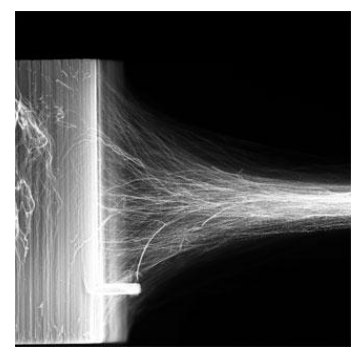

Fig. 1. A carbon nanotube sheet being drawn from a cluster of nanotubes.

\section{B. Other Nanoparticles}

Nanostructured materials may be defined as those materials whose structural elements-clusters, crystallites or molecules-have dimensions in the 1 to $100 \mathrm{~nm}$ range. Though not much different than those of CNTs, a couple of them can be physically and chemically enhanced to suit the needs of an on-field soldier. For example, nanophase 
ceramics, unlike conventional ceramics are ductile and more sustainable than conventional metals in harsh environments. Nanoparticle coating can improve toughness and resistance to wear, corrosion, oxidation and cracks. The coupling between several metallic nanoparticles can increase phenomena like scattering, absorption, luminescence, or Raman scattering. For instance, while yttria, a conventional ceramic, is opaque, its nanophase counterpart can be made transparent. The tiny grains in such nano structured materials can block (absorb or scatter) ultraviolet rays because of their much shorter wavelengths. Advanced polymeric nanocoatings show promise in making sensors that are both highly selective and sensitive to parts per trillion (see Fig. 2).

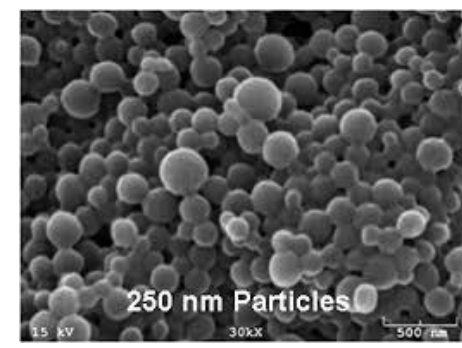

Fig. 2. Magnified image of nanoparticles.

\section{APPLICATIONS OF NANOTECH FOR BATTLEFIELD EFFICIENCY}

Nanotech has a variety of applications in the defense sector. Battlefield efficiency can be obtained by fulfilling the ideal requirements of the basic necessities required in every warcombat suits, artillery, transportation, logistics, and hazard detection and prevention. The application of the respective nanomaterial can increase the effectively reduce the mortality rates of the soldiers while simultaneously increasing the mortality rates of the opponents.

\section{A. Combat Suits}

The typical combat suits can comprise of:

1) Lightweight protective clothes-bullet-proof and with increased mobility.

2) Adaptive suits- improved thermal control, switchable camouflage

3) Microsensors, readers, displays, radio transmitters

- Stiff exoskeletons for added protection

The aforementioned properties of CNTs- hardness, strength, thermal properties can be useful for tailoring lightweight, bullet-proof combat suits. Body armor made of CNT is 117 times stronger than steel [2], [3]. The thermal adaptability of the body can be monitored via nanosensors embedded into the suits thus accordingly manipulating its temperature. Nanocoatings can be used to enhance camouflaging.

Nanotube-enhanced conductive plastics can be used in nanosensors for helmets. They enable electrostatic dissipation and electromagnetic-wave shielding to prevent interference in battlefield communication systems.

As has been suggested by Wang et al. in his paper [4], nanoparticle silica 'paint' can be used to create surface with anti-fog properties. Nanoparticles, due to their optical properties can be used to filter specific wavelengths of light thus keeping harmful radiations at bay. This would allow for self-cleaning smart glasses to better control heat, UV dispersion, thus increasing eye safety and enhancing vision, especially in regions prone to extreme high temperatures [5].

Nanosensors can also be used to monitor the health of soldiers and to track their positions thus resulting in prompt medical aids and search-and-rescue operations. They could also be used to monitor the surroundings of the soldier, and accordingly enable selective camouflaging, thus eliminating the need for different body armors for different environments.

\section{B. Artillery}

The requisite components for better armaments are remote, unmanned guidance, adaptive camouflaging, precision targeting, biometric sensors (for personalized access), minimum weight, optimum impact damage and a virtually undetectable signature.

The unmanned guidance, precision targeting and minimum signature can be taken care of by nanosensors which operate real time to adapt to the environment and acquire the exact position of the target [6], [7].

High energy nanocomposite materials can be used for high impact ammunitions.

CNTs can be used to ensure a lightweight, yet rigid structure for the main body of the weapon.

Even nanopoisons are being considered as a potential weapon against soldiers. Discreet amounts of it could harm the soldiers' health enough to incapacitate them before or during a war. These could be virtually undetectable and contagious [2].

Though still at a theoretical level, self-replicating nanobots are being considered as weapons of mass-destruction.

The traditional military hesitation towards biological agents for fear that their own forces and population could be infected, too, can be eradicated by the new developments in nanotech towards DNA sampling and gene therapy. The recent emergence of CNTs as potential components for DNA recognition and alternatives for gene carrier systems have made them eligible for selective action. This will render the soldiers better protection against biological and chemical weapons and eliminate their hesitation against using them [3].

\section{Transport: Vehicles, Aircrafts, Submarines}

The basic facilities that should be accommodated by all vehicles are [8]:

- Stealth radar

- Adaptive camouflaging

- Biometric id to prevent sabotage

- Remote guidance

- Real time satellite updates for navigation and GPS

- Directional RF(radio- frequency) communication enabled by micro antenna arrays for reduced power and signature

- Shock absorbent armour

- Rigid/flexible suspension (terrain-dependant) in case of vehicles

The automobile alternators and submarines can be made quieter by the use high-power magnets manufactured from nanocrystals. According to magnetohydro dynamics (MHD), 
the concept of using the conductivity of sea water based on the principle of Fleming's left-hand rule to enable noiseless propulsion of submarines can be exploited with the help of superconductive magnets. Using nanocrystals to manufacture such magnets might enable quieter, stealthier submarines. Nanocrystals in nanocoatings can also be utilized to enable adaptive camouflaging.

Reportedly, the use of nanostructured materials (e.g, CNT) in aircraft can increase structural lifetime by as much as $300 \%$, due to their strength, hardness and weight reduction properties. Florida Advanced Center for Composite Technologies (FAC2T) predicts buckypaper (created from carbon nanotubes, ultrathin, electrically conducting and 10 times lighter than steel while still being 250 times stronger) will prove applicable as a high-current protective film for the exteriors of airplanes which would allow lightning strikes to flow around a plane and dissipate without damaging it.

Nanophaseceramics can be moulded into automobile parts which are cheaper and more durable in harsh conditions and corrosive atmospheres than conventional metal parts. Nanocoatings on engine cylinders can reduce heat loss, corrosion and improve fuel combustion.

Nanoparticle reinforced polymers can make engine tanks in army vehicles, submarines and aircrafts $40 \%$ to $60 \%$ lighter [4].

With added nanotubes, plastics, such as polyaniline, can conduct better than copper. Replacing heavy copper wires in aircrafts with such lightweight conducting plastic wires can result in weight reduction which will have positive implications on speed, range, fuel consumption and maneuverability (see Fig. 3).

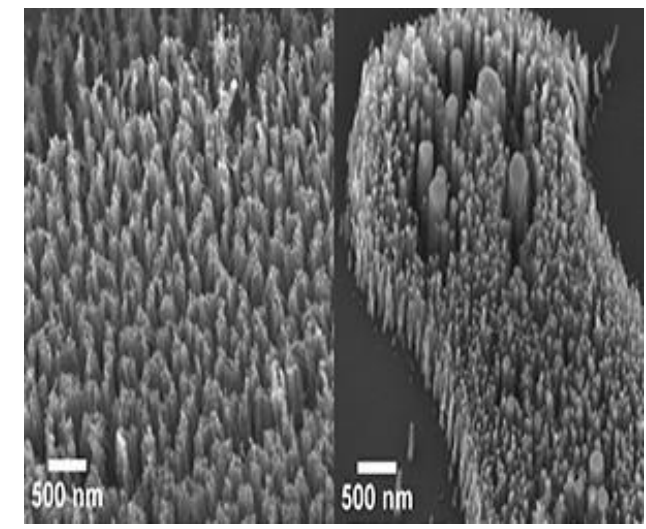

Fig. 3. Nanoparticle reinforced polymers can make engine tanks in army vehicles, submarines and aircrafts $40-60 \%$ lighter.

\section{Logistics}

Logistics is the management of the flow of resources between the point of origin and the point of consumption in order to meet some requirements. The logistics of physical items usually involves the integration of information, flow, material handling, production, packaging, inventory, transportation, warehousing, and often security.

Logistics in military systems are expected to have [8]:

- Lightweight containers

- Shock-absorbing materials

- Alarm for mechanical or chemical deficiencies

- GPS trackers

- Artificial electronic nose to detect gases and bacterial or

\section{chemical decontamination}

- Biometric sensors for personalized access and tamper-proof security.

Nanocomposite plastics can be used for the manufacture of rigid, lightweight containers.

Multifunctional nanosensors embedded into the containers could be used for alarms, GPS trackers, detection of foreign particles and biometric ids.

\section{E. Hazard Detection and Prevention}

There is a vast potential for nanosensors in this area. Nanosensors would give warning against biological and/or chemical weapons. They can also be useful for monitoring the physical health of the soldiers. They can be used to detect any poisonous substances (nanopoisons), which would otherwise be virtually undetectable [7].

Built-in protective filters can filter harmful nanoparticles thus ensuring the immunity of soldiers against biological and chemical agents.

The highly sensitive electrical conductance of semiconducting nanotubes can be used to constantly scan the conditions of the various logistics and artillery thus reducing the risk of any tampering or sabotage and enabling early detection of any defects.

Nanoparticles can also be used in water-purifying pills to reduce the risk of illness due to water contamination [2] (see Fig. 4).

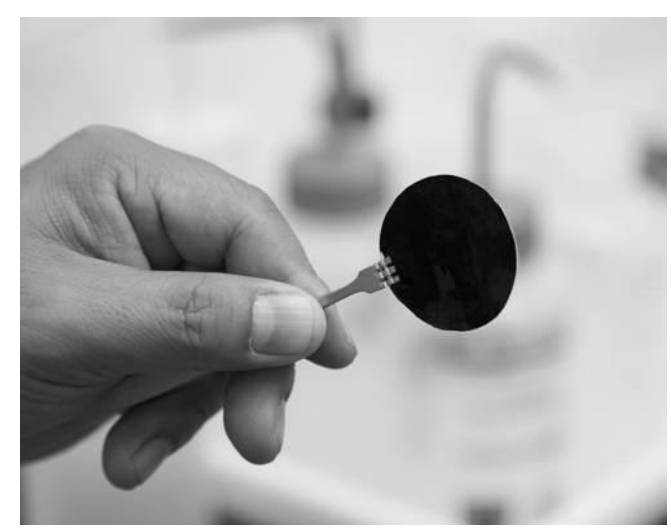

Fig. 4. Nanotech 'second skin' could protect soldiers from chemical threats [9].

\section{PROS AND CONS OF THEIR IMPACT}

The influence of nanotech in the defense sector will undoubtedly be greatly advantageous to the soldiers. Not only will it result in lower mortality rates, it will also enable the advent of a new generation of artificial intelligence. The use of the potent, and infinitely more powerful nanotech-based weapons could encourage better battlefield efficiency [10] (see Fig. 5).

However, nanotech may also pose grave risks in the form of invasion of privacy, increased pollution and displacement of soldiers [11]. The use of nanopoisons can, over time, result in permanent physical damage and deformity and also carries the risk of mutation in extreme cases.

Diffusion of these systems and technology to criminals and terrorists could open up new options for crime and misdemeanors.

Self-replicating nanobots, if mishandled, could result in 
the disruption of an entire society [11].

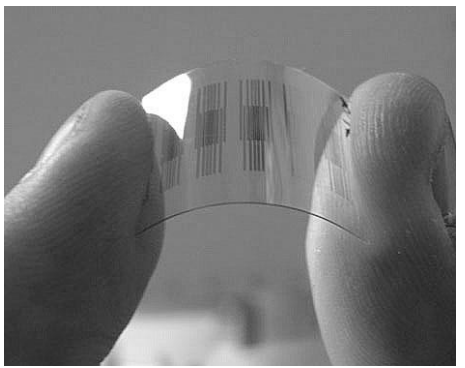

Fig. 5. Nanosensor attached to cell surfaces to eavesdrop on chat real-time [12].

\section{CONCLUSION}

Warfare has been an unavoidable aspect of human survival. The onset of technology in the defense sector, though resulting in better warfare techniques with the intention of reducing the mortality rates and the grievances associated with them, has ironically created more lethal weapons for their destruction. Nanotechnology has further solidified the need for safer yet more high-impact weaponry due to the unique properties associated with nanomaterials. In the right hands, this could enable a better tomorrow. In the wrong ones, there might not be a tomorrow.

\section{ACKNOWLEDGMENT}

The author would like to thank the Director and the faculty of BITS Pilani, Dubai Campus for their valuable guidance and support.

\section{REFERENCES}

[1] P. Brian et al., "Unmanned Aerial Vehicles: The Force Multiplier of the 1990s," Airpower Journal, pp. 41-54, 1991.

[2] A. Tiwari, "Military nanotechnology".

[3] J. Altmann, "Limiting Military Uses of Nanotechnology and Converging Technologies," Marburg, Germany, 2005, pp. 13-15.

[4] J. Wang and P. J. Dortmans, "A review of selected nanotechnology topics and their potential military applications," Defense science and technology organisation salisbury (australia) systems sciences lab, 2004.

[5] Carbon Nanotube Films Transparent Conductive Films. (Dec. 13, 2013). Unidym. N.p., n.d. Web. [Online]. Available: http://www.unidym.com/index.html

[6] P. B. Ruffin, "Nanotechnology for missiles," Integrated Optoelectronic Devices 2004, International Society for Optics and Photonics, 2004.

[7] J. V. Guill, "The Nose Knows: Developing Advanced Chemical Sensors for the Remote Detection of Improvised Explosive Devices in 2030," Air command and staff coll maxwell afb al, 2009.

[8] F. Simonis and S. Schilthuizen, "Nanotechnology: Innovation Opportunities for Tomorrow's Defense," TNO Science and Industry, 2006.

[9] Nbc News Technology. (13 Dec. 2013). NBC News. N.p., n.d. Web. [Online]. Available: http://www.nbcnews.com/technology/nanotech-second-skin-could-pro tect-soldiers-chemical-threats-1C7291458

[10] Nanomagazine. (13 Dec. 2013). Nanomagazine. N.p., n.d. Web. [Online]. Available: http://www.nanomagazine.co.uk/index.php?option=com_content

[11] A. Jürgen. "Military uses of nanotechnology: perspectives and concerns," Security Dialogue, vol. 35, no, 1, 2004, pp. 61-79.

[12] Eavesdropping on Cells' Chats with Nano-sensors TopNews. (13 Dec. 2013). Eavesdropping on Cells' Chats with Nano-sensors TopNews. N.p., n.d. Web. [Online]. Available: http://www.topnews.in/usa/eavesdropping-cells-chats-nano-sensors-2 11550

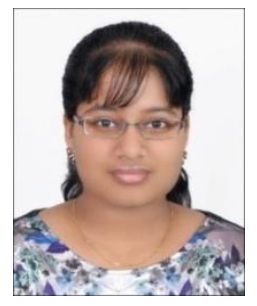

Mahidhara Utsav was born in NaviMumbai, India on October 2, 1995. She is currently a freshman in BITS Pilani Dubai Campus and is pursuing B.E. (Hons) in electronics and electrical engineering.

She has completed a one-month internship in ElectroRAK, a leading MEP solutions provider company in the UAE. During her stint in ElectroRAK, she has worked in the Design and the Estimation Departments, among others. Her first review paper on CNTs in Nanocommunication and Their Application in the Health Sector has been selected for presentation in the 4th Annual International Conference on Advances in Biotechnology (BIOTECH 2014), Dubai, UAE.

Ms. Mahidhara is currently a member of IEEE and ASME. 